\title{
Translational Neurocritical Care Research: Advancing Understanding and Developing Therapeutics
}

\author{
J. Claude Hemphill III ${ }^{1,2}$ (D) $\cdot$ Michael L. James ${ }^{3}$ \\ Published online: 18 May 2020 \\ (C) The American Society for Experimental NeuroTherapeutics, Inc. 2020
}

Neurocritical Care is a relatively young discipline. While patients with traumatic brain injury, stroke, and seizures have needed care since time immemorial, organized neurointensive care units, formal provider training, and a coordinated body of neurocritical care science have only been around for about 30 years. Several advances occurring in the 1990s, specifically intravenous recombinant tissue plasminogen activator use after acute ischemic stroke [1] and advancement of neurological monitoring, compelled the creation of guidelines and procedures for the care of patients with acute neurological injury [2, $3]$, development of highly specialized units, and training of qualified professionals [4, 5]. Early development of neurocritical care focused on aspects related to clinical patient care: increasing numbers of practitioners with formal fellowship training and organizing neurocritical care programs within hospitals to cohort and standardize care. Clinical trials for many aspects of neurocritical care were few and far between, but observational patient-oriented research developed quickly. This notion of the "bedside" informing relevant questions that could and should be investigated at the "bench" became part of the ethos of the ongoing development of neurocritical care, even as there remained a relative paucity of translatable findings. Fundamental concepts, such as the relative roles of primary and secondary brain injury in stroke, trauma, seizures,

Electronic supplementary material The online version of this article (https://doi.org/10.1007/s13311-020-00867-w) contains supplementary material, which is available to authorized users.

J. Claude Hemphill, III

claude.hemphill@ucsf.edu

1 Departments of Neurology and Neurological Surgery, University of California, San Francisco, CA, USA

2 Department of Neurology, Zuckerberg San Francisco General Hospital, Building 1, Room 101, 1001 Potrero Avenue, San Francisco, CA 94110, USA

3 Departments of Anesthesiology and Neurology, Duke University, Durham, USA and brain infections, became pillars upon which future work would be anchored.

Concurrent with and even preceding this era, there was substantial and robust laboratory-based discovery science using a variety of preclinical models of cellular mechanisms of injury in focal ischemia, traumatic brain injury, and intracranial hemorrhage. Great enthusiasm for this bench-tobedside model yielding treatments resulted in elucidation of injury cascades. These discoveries led to numerous clinical trials of pharmacological agents targeting specific pathways such as excitotoxicity, cellular calcium influx, and free radical membrane injury. As we all recognize now, none were successful in human clinical trials, and dissecting the "failure of neuroprotection" has been a repeated pastime [6, 7]. Perhaps the greatest lesson from these early failures was recognition that brain mechanisms are complicated, therapeutic development will not be easy, and more fluid integration of "bedside and bench" techniques, methodologies, and discussions are necessary [8].

While relevant science was being published and presented in venues related to overall critical care medicine, neurosurgery, and neurology, the formation of the Neurocritical Care Society (NCS) in 2003 was a foundational step towards coalescing the science into a more cohesive and thematically focused body. NCS is the first and largest international society with the mission of advancing patient care, research, and education for neurocritical care. The annual meeting became a place for clinicians and scientists to interact, present relevant science, define the most compelling questions, and develop collaborations. Over the next decade, the science developed from principally clinically focused to (re)embracing the need for translational scientists who understood the bedside and could investigate at the bench. Probably most of all, there came the recognition that neurocritical care is inherently multidisciplinary and that investigators and clinicians are more defined by the conditions they study and treat rather than their training lineage. Specifically encouraging and developing young investigators in translational neurocritical care research 
has been increasingly prioritized, as can happen when a clinical field matures in its organization.

This brings us to today and our Neurocritical Care-focused issue of Neurotherapeutics. When approached with the idea of putting together an issue that addresses translational neurocritical care science, we were excited by the opportunity to highlight a range of questions that have become increasingly relevant to clinical care and development of therapeutics for the field. Perhaps even more importantly, we were excited about the opportunity to learn from many of the emerging leaders in neurocritical care translational science regarding where we are and want to go. Notably all corresponding authors for these manuscripts are junior or mid-career investigators who have emerged from the evolution of neurocritical care to advance its translational science.

Several themes develop throughout this issue of Neurotherapeutics: therapeutic development from enhanced understanding of inflammation and immune modulation, cell injury/death, and harnessing endogenous responses; advent of enhanced monitoring and therapeutic device development; and use of advancing technologies related to genetics, genomics, and informatics. The spectrum of neurocritical care conditions is not limited to stroke and neurotrauma. For example, Mazeraud et al. [9] discuss sepsis-associated encephalopathy as a particularly common occurrence in general critical care contributing to increased morbidity. Relatedly, systemic inflammation may impact blood-brain barrier (BBB) integrity with the limbic system being specifically vulnerable. Kolls et al.'s [10] examination of NMDA receptor-related autoimmune encephalitis highlights translation of a previously mysterious condition now made clear with subsequent direction for treatment. Following on the themes of immune modulation, discussion of the local and peripheral immune response in the context of stroke by Zera and Buckwalter demonstrates the "two-way" communication in which patients may have increased vulnerability to systemic infection such as pneumonia in the setting of a primary neurological condition such as acute stroke [11]. Similarly, Coulibaly and Provencio review how immune responses mediate vascular and neural changes after subarachnoid hemorrhage with the ultimate effector cells being parenchymal brain and peripheral immune cells [12] These acute central responses to injury may have long-term neurological effects, as summarized by Akamatsu and Hanafy in the setting of neurocognitive decline after traumatic brain injury [13]. "Resilience" to such injuries, as a new and potentially clinically applicable type of neuroprotection distinct from prior concepts, is modeled in studies of hibernation in animals such as arctic ground squirrels as detailed by Singhal et al. [14]. In fact, endogenous responses to injury may play a role in neurorestorative mechanisms. James et al. provide a potential framework for harnessing the brain's inherent responses using apolipoprotein $\mathrm{E}$ as a candidate therapeutic [15]. Likewise, Lattanzi et al. examine matrix metalloproteinases' role in membrane integrity after intracerebral hemorrhage, explaining some unique aspects of secondary brain injury for this condition, and suggest potential therapeutics [16]. Finally, Sugimoto and Chung consider the value of cortical spreading depression as an emerging therapeutic target and biomarker after subarachnoid hemorrhage, assessing the generalizability of existing therapeutic trials and ways to define its relationship with functional outcome [17].

In addition to therapeutics, neurocritical care has witnessed a font of monitoring and device development through translational work. Saadoun and Papadopolous review the monitoring of spinal cord blood flow after acute injury, revealing distinct blood flow patterns (e.g., local steal and diastolic ischemia) that may enable optimized and individualized spinal cord perfusion therapy [18]. Also, Alambyan et al. discuss noninvasive metabolic imaging as a tool for molecular level energy exchange to guide targeted management in a diverse array of neuropathology [19]. Additionally, Hosseini et al. assess potential combination therapies (e.g., pharmacotherapy, perfusion/oxygenation targets, and pre/post-conditioning) with targeted temperature management after hypoxicischemic brain injury [20].

Finally in this issue, we are given insight into how advances in genetics, genomics, and computing capabilities are providing new opportunities to better study how our patients respond to disease and how we can integrate the data we have available (and new data to come). Interestingly, as described by Myserlis et al., translational genomics can be utilized for both forward and reverse translation regarding many of the issues of inflammation, the BBB, and cellular vulnerability across conditions [21]. Acosta et al. elucidate how large collaborations, public access to data, reproducible science, and innovative analytical methods have exponentially increased the pace of discoveries related to neurocritical care and how new treatments may be developed through supportive genetic evidence [22]. Finally, how to bring data together, whether from electronic health records or bedside "signals" in use today such as intracranial pressure waveforms, and apply machine-learning techniques to provide insights not immediately apparent to bedside clinicians is described by Foreman [23].

One obvious overall conclusion from this issue of Neurotherapeutics is that neurocritical care translational science has evolved greatly since the origins of organized neurocritical care. The bedside-to-bench-to-bedside model that is evidenced by the authors of these manuscripts resonates as a pathway for developing new pharmacological treatments, clinical prediction and decision support, and guidelines. We hope you enjoy and learn from the manuscripts in this issue as we work together to expand collaborations and prioritize developing young investigators with the goal of improving treatments for our patients. 
Required Author Forms Disclosure forms provided by the authors are available with the online version of this article.

\section{References}

1. The National Institute of Neurological Disorders and Stroke rt-PA Stroke Study Group. Tissue plasminogen activator for acute ischemic stroke. N Engl J Med 1995;333(24):1581-7.

2. Bullock R, Chesnut RM, Clifton G, Ghajar J, Marion DW, Narayan RK, et al. Guidelines for the management of severe head injury. Brain Trauma Foundation. Eur J Emerg Med 1996;3(2):109-27.

3. Moheet AM, Livesay SL, Abdelhak T, Bleck TP, Human T, Karanjia N, et al. Standards for Neurologic Critical Care Units: A Statement for Healthcare Professionals from The Neurocritical Care Society. Neurocrit Care 2018;29(2):145-60.

4. Diringer MN, Edwards DF. Admission to a neurologic/ neurosurgical intensive care unit is associated with reduced mortality rate after intracerebral hemorrhage. Crit Care Med 2001;29(3): 635-40.

5. Morris NA, Czeisler BM, Sarwal A. Simulation in Neurocritical Care: Past, Present, and Future. Neurocrit Care 2019;30(3):522-33.

6. Gladstone DJ, Black SE, Hakim AM, for the Heart and Stroke Foundation of Ontario Centre of Excellence in Stroke Recovery. Toward wisdom from failure: lessons from neuroprotective stroke trials and new therapeutic directions. Stroke. 2002;33(8):2123-36.

7. Loane DJ, Faden AI. Neuroprotection for traumatic brain injury: translational challenges and emerging therapeutic strategies. Trends Pharmacol Sci 2010;31(12):596-604.

8. Warner DS, James ML, Laskowitz DT, Wijdicks EF. Translational research in acute central nervous system injury: lessons learned and the future. JAMA Neurol 2014;71(10):1311-8.

9. Mazeraud A, Righy C, Bouchereau E, Benghanem S, Bozza FA, Sharshar T. Septic-Associated Encephalopathy: A Comprehensive Review. Neurotherapeutics 2020;2.
10. Kolls B, Yasmin A O'Keefe YA, Sahgal AK. Autoimmune Encephalitis: NMDA Receptor Encephalitis as an Example of Translational Neuroscience. Neurotherapeutics 2020;2.

11. Zera KA, Buckwalter MS. The Local and Peripheral Immune Responses to Stroke: Implications for Therapeutic Development. Neurotherapeutics 2020;2.

12. Coulibaly AP, Provencio JJ. Aneurysmal Subarachnoid Hemorrhage: An Overview of Inflammation-Induced Cellular Changes. Neurotherapeutics 2020;2.

13. Akamatsu Y, Hanafy KA. Cell Death and Recovery in Traumatic Brain Injury. Neurotherapeutics 2020;2.

14. Singhal NS, Sun CH, Lee EM, Ma DK. Resilience to Injury: A New Approach to Neuroprotection? Neurotherapeutics 2020;2.

15. James ML, Komisarow JM, Wang H, Laskowitz DT. Therapeutic Development of Apolipoprotein E Mimetics for Acute brain Injury: Augmenting Endogenous Responses to Reduce Secondary Injury. Neurotherapeutics 2020;2.

16. Lattanzi S, Di Napoli M, Ricci S, Divani AA. Matrix Metalloproteinases in Acute Intracerebral Hemorrhage. Neurotherapeutics 2020;2.

17. Sugimoto K, Chung DY. Spreading Depolarizations and Subarachnoid Hemorrhage. Neurotherapeutics 2020;2.

18. Saadoun S, Papadopoulos MC. Targeted Perfusion Therapy in Spinal Cord Trauma. Neurotherapeutics 2020;2.

19. Alambyan V, Pace J, Sukpornchairak P, Yu X, Alnimir H, Tatton R, et al. Imaging Guidance for Therapeutic Delivery: The Dawn of Neuroenergetics. Neurotherapeutics 2020;2.

20. Hosseini M, Wilson RH, Crouzet C, Amirhekmat A, Wei KS, Akbari Y. Resuscitating the Globally Ischemic Brain: TTM and Beyond Neurotherapeutics 2020;2.

21. Myserlis P, Radmanesh F, Anderson CD. Translational Genomics in Neurocritical Care: A Review. Neurotherapeutics 2020;2.

22. Acosta JN, Brown SC, Falcone GJ. Genetic Variation and Response to Neurocritical Illness: A Powerful Approach to Identify Novel Pathophysiological Mechanisms and Therapeutic Targets. Neurotherapeutics 2020;2.

23. Foreman B. Integrating and Using Big Data in Neurocritical Care. Neurotherapeutics 2020;2.

Publisher's Note Springer Nature remains neutral with regard to jurisdictional claims in published maps and institutional affiliations. 